\title{
Optimal Control of Electromagnetic Suspension EMS System
}

\author{
Anan Suebsomran*
}

Department of Teacher Training in Mechanical Engineering, King Mongkut's University of Technology North Bangkok, 1518 Pibulsongkram Rd.,Bagsue, 10800, Bangkok, Thailand

\begin{abstract}
This paper presents the controller design of magnetic levitation application. The highly nonlinear electromagnetic suspension EMS system is hard and limited system control subjected to prescribed stability of system. Due to the nonlinear dynamics of system, the linearization of the nonlinear EMS plant is described by linear model. An attraction force about the prescribed nominal operating point of current and air gap positioning is chosen for linearization by a nominal operating point. Optimal control is applied for controlling nonlinear dynamics of EMS plant. Linear quadratic regulator (LQR) controller applies for this control objective. The system stability is proofed by using Lyapunov's method. From the results the reference of air gap position can be tracked with the desired nominal operating control as shown in simulation and practical manner.
\end{abstract}

Keywords: Electromagnetic suspension EMS system, linear quadratic regulator (LQR), linearization, lyapunov's method, optimal control, riccati equation.

\section{INTRODUCTION}

Electromagnetic suspension EMS system is significantly applied in advanced technology such as ground transportation, magnetic bearing and other applications. Control of electromagnetic suspension EMS system is a key technology but has some difficulties due to its nonlinearity, instability, and uncertainty. To overcome the problems mentiond. This research focuses on designing the control system for increasing the performance of EMS system. Normally many researchers applied the nonlinear control for EMS system. From references [1] proposed nonlinear state and outputfeedback $H_{\infty}$ controllers to suppress guide way induced disturbances. From experiment result, air-gap position can be controlled at $4 \mathrm{~mm}$ at nominal operating point. [2] also proposed the designed linear quadratic state feedback regulator. It can maintain the closed-loop stability in the presence of some certain actuator failures. The control of such a system is applied to fault control application of electromagnets suspension model of maglev train. The simulation study of the design is done by numerical study of magnetic levitation model in linear model. [3] presented the results on the robust stabilization of a class of feedback linearized nonlinear single-input/single-output (SISO) systems with parametric uncertainty. They implemented of nonlinear feedback linearizing control for an electromagnetic suspension EMS system. Comparison on the technique of feedback linearization and classical state feedback control by using linearization with small perturbation is demonstrated by practical experiment. They concluded that feedback linearization control yielded

*Address correspondence to this author at the Department of Teacher Training in Mechanical Engineering, King Mongkut's University of Technology North Bangkok, 1518 Pibulsongkram Rd., Bagsue, 10800, Bangkok, Thailand; Tel: +66 87020 3127; Fax: +662 587 8261; E-mail: asr@kmutnb.ac.th the stable control of tracking at air gap reference, while the classical state feedback control using linearization at small perturbation could not keep system stable. The model of dynamics and control of electromagnetic suspension is described by [4]. And [5] also proposed the applied magnetic levitation for educational purpose. They described the operation of a pulse width modulation converter in a magnetic suspension system. The pulse width-modulated (PWM) converter illustrates modern principles of power electronics, such as PWM control, current-mode control, averaged and linearized models of switched-mode converters, and power supply design. The experimental system shown the levitation control with diameter $6 \mathrm{~cm}$ and weight $0.8 \mathrm{~kg}$ of a metallic sphere. [6] applied the laser displacement measurement for the feedback control of air gap of magnetic levitation and suspension system. Then system is increased the accuracy and smoothness of control output signal due to the high resolution of feedback measurement device.

\section{MATHEMATICAL MODEL}

Schematic diagram of electromagnetic suspension EMS system of prototype development is illustrated in Fig. (1) for a single-degree-of-freedom suspension system prototype with a controlled dc electromagnet. The reaction surface of the rail is fixed, suspension magnet and payload suspense under the fixed ferromagnetic track. The flux are generated by the electromagnet coil and passed though the fix ferromagnetic track, since the coil inductance is calculated by (1).

$$
\mathrm{L}(\mathrm{z})=\frac{\mathrm{N}}{\mathrm{i}(\mathrm{t})} \Phi_{T}=\frac{N^{2}}{R_{T}}
$$

Where $\mathrm{N}$ is the number of turns of the coil, $\mathrm{i}(\mathrm{t})$ is the current to the coil, $\Phi_{T}$ is total flux of magnetic circuit, and $R_{T}$ is the total reluctance of magnetic circuit. 


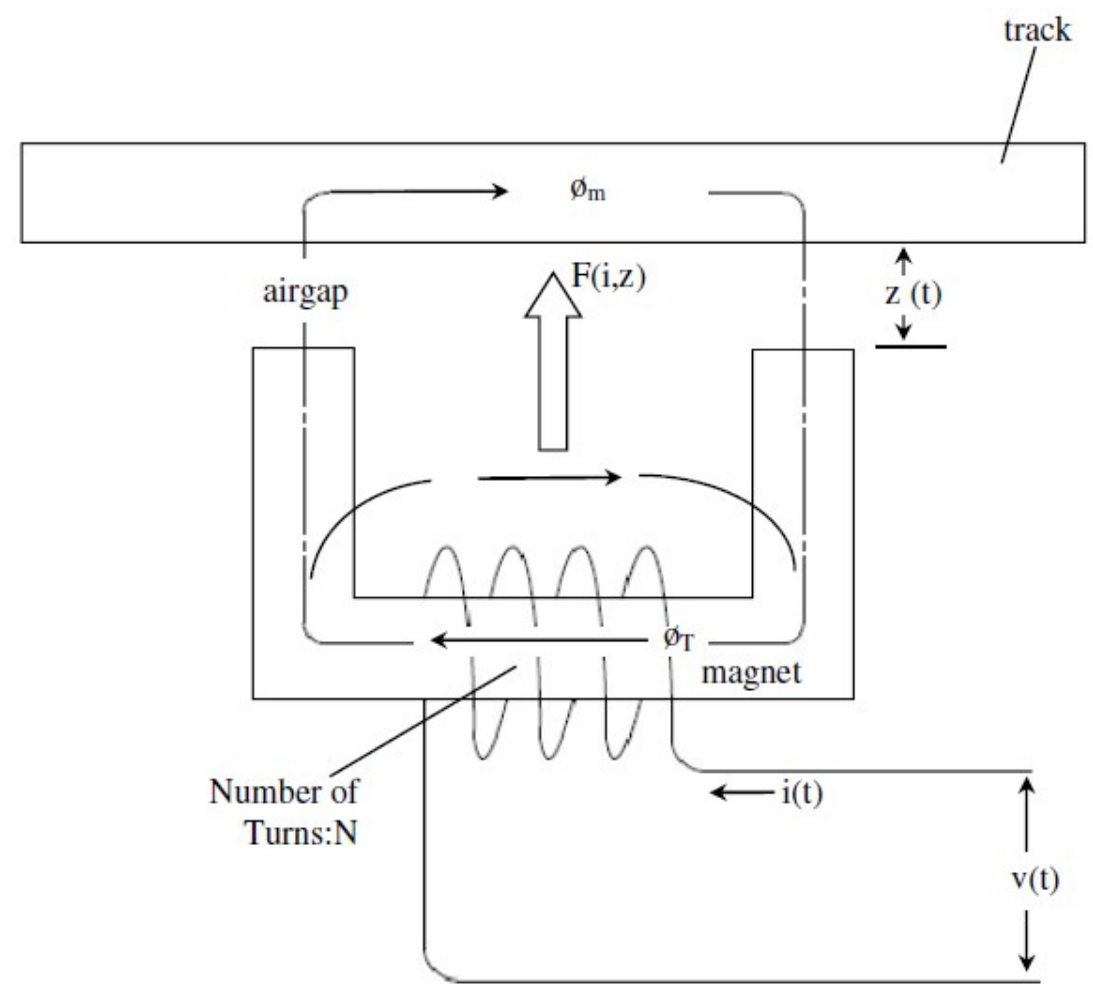

Fig. (1). Electromagnetic suspension EMS system of ferromagnetic guide track and electromagnet coil configuration.

If the reluctance of magnetic core is neglected when applied to two air-gaps, then the coil inductance is expressed as (2) with total length of magnet core equal to $2 z(t)$.

$\mathrm{L}(\mathrm{z})=\frac{\mu_{0} N^{2} A}{2 \mathrm{z}(\mathrm{t})}$

Where $\mu_{0}$ is the permeability in free space, $z(t)$ is the airgap between magnet pole and ferromagnetic guide-way. The force of attraction between magnet coil and ferromagnetic rail is given by (3)

$\mathrm{F}(\mathrm{i}, \mathrm{z}, \mathrm{t})=-\frac{\mathrm{d}}{\mathrm{dt}}\left[\frac{1}{2} L(z) i(t)^{2}\right]$

Inductance form (2) is substituted into (3), we can obtained the force attraction by (4)

$\mathrm{F}(\mathrm{i}, \mathrm{z}, \mathrm{t})=\frac{\mu_{0} N^{2} A}{4}\left[\frac{i(t)}{z(t)}\right]^{2}$

Using the notations given in Fig. (1), the vertical dynamics is described by [2] as its motion is governed by the differential dynamic equations of motion of electromagnetic suspension system in (1), (2) and (3) respectively, which concerns in dynamic motion of levitated object.

$m \frac{d^{2} z(t)}{d t}=-F(i, z, t)+f_{d}+m g$

Where $F(i, z, t)$ is the electromagnetic force attraction. $f_{d}$ is force disturbance. By substituting $-\frac{\mu_{0} N^{2} A}{4}\left[\frac{i(t)}{z(t)}\right]^{2}$ into (1), the equations of motion can be rearranged in (5) and (6) respectively.

$m \frac{d^{2} z(t)}{d t}=-\frac{\mu_{0} N^{2} A}{4}\left[\frac{i(t)}{z(t)}\right]^{2}+f_{d}+m g$

The relationship between the time-varying voltage $v(t)$ across an inductor with inductance $L$ and the time-varying current $i(t)$ passing through it is described by the differential equation.

The electromotive force $\Theta$ is

$\Theta=N i$

Thus the flux $\Phi_{f c}$ produced through the electromagnet coil is

$\Phi_{f c}=\frac{\Theta}{R_{T}}$

Where $R_{T}$ is the total reluctance of the electromagnet circuit which comprise of the reluctance of magnet core, levitated object, air gap, leakage fluxes. The reluctance is the function of geometrical and magnetic parameter by [7]. The flux linkage $\psi$ of electromagnetic coil is expressed in (9).

$\psi=N \Phi_{f c}$

Electromagnetic suspension model of magnetic coil with $N$ turns is applied voltage $v(t)$ across the terminal of coil and the current $i(t)$ produces the magnetic field in air gap be- 
tween magnet core and fixed ferromagnetic track. The reluctances comprise of the reluctance of magnet core $R_{f c}$, the reluctance of levitated object $R_{f o}$, the reluctance of air gap $R_{g}$, and the reluctance of leakage fluxes $R_{l}$. The reluctance is the function of geometrical and magnetic parameter as expressed in (10).

$$
R_{T}=R_{f c}+\frac{R_{l}\left(R_{g}+R_{f o}\right)}{R_{l}+R_{g}+R_{f o}}
$$

Where

$R_{f c}=\frac{l_{f c}}{\mu_{o} \mu_{r} A_{f c}}$ is reluctance of the core.

$R_{f o}=\frac{l_{f o}}{\mu_{o} \mu_{r} A_{f o}}$ is reluctance of the levitated object.

$R_{g}=\frac{z}{\mu_{o} A_{g}}$ is reluctance of air gap.

$R_{l}=\frac{l_{l}}{\mu_{o} \mu_{r} A_{l}}$ is leakage reluctance.

From flux linkage $\psi$ of (9), the Faraday law is expressed by (11) and (12).

$$
\frac{d}{d t} \psi=-R i(t)+v(t)
$$

Or

$$
v(t)=R i(t)+\frac{d}{d t} \psi
$$

Where $R$ is an electrical resistance of circuit. $i(t)$ is the current of circuit. $v(t)$ is the applied voltage input to circuit. Flux linkage $\psi$ is the function of current and position of levitated object and it can be expressed by the relationships in partial differentiation as described in (13).

$$
\frac{d}{d t} \psi=\frac{\partial \psi}{\partial i} \frac{d i}{d t}+\frac{\partial \psi}{\partial s} w=L(z) \frac{d i}{d t}+\frac{\partial L(z)}{\partial z} w i
$$
(14).

Substitutes flux linkage into (13), it can be expressed as

$$
\frac{d i}{d t}=\frac{1}{L(z)}\left(-R i(t)-\frac{\partial L(z)}{\partial z} w i+v(t)\right)
$$

Where $w$ is the velocity of levitated object, which is $\dot{z}$. The differentiation of instantaneous current with respect to time caused by the voltage induce the coil as (14) to produce the magnet field by inductance $\mathrm{L}$ between fix ferromagnetic guide way and magnet core and coil. The inductance is the function of position of levitated object which is found in (2). By substituting (2) into (14), it results in the electrical circuit of electromagnetic with applied input voltage in (15) across the circuit as indicate the state of current can be found as follows; $\frac{d i(t)}{d t}=\frac{i(t)}{z(t)} \frac{d z(t)}{d t}-\frac{2}{\mu_{0} N^{2} A} z(t)(R i(t)-u(t))$

From the differential equations of motion of nonlinear electromagnetic suspension system in (1) and (15), it converts to nonlinear state-space form in (16). The state vector can be defined as $x(t)=(z(t) \quad \dot{z}(t) \quad i(t))^{T} \in R^{n}, n=3$. The state variables, $x_{1}=z(t)$ and $x_{2}=\dot{z}(t)$, are the position and velocity of electromagnetic suspension coil respectively. And $x_{3}=i(t)$ is the current of electromagnetic coil.

$$
\begin{aligned}
& \dot{x}_{1}=x_{2}(t) \\
& \dot{x}_{2}=-\frac{\mu_{0} N^{2} A}{4 m}\left(\frac{x_{3}(t)}{x_{2}(t)}\right)^{2}+f_{d}+g \\
& \dot{x}_{3}=\frac{x_{2}(t) x_{3}(t)}{x_{1}(t)}-\frac{2 R}{\mu_{0} N^{2} A} x_{1}(t) x_{3}(t)+\frac{2 x_{1}(t)}{\mu_{0} N^{2} A} u(t) \\
& y=x_{1}(t)
\end{aligned}
$$

Where $x_{1}(t)$ is vertical gap. $x_{2}(t)$ is vertical velocity. $x_{3}(t)=i(t)$ is the magnet current.. $u(t)=v(t)$ is the applied voltage. $N$ is the number of turns of coil around the magnet. $m$ is total mass. $A$ is the pole area of magnet. $\mu_{0}$ is the constant permeability of free space. $R$ is the coil resistance. $g$ is the gravity constant. $z_{r e f}$ is the reference air gap. $f_{d}$ is the force disturbance. Finally the state-variable equations can be obtained with subject to initial condition as illustrated in (17) in matrix form.

$$
\left(\begin{array}{l}
\dot{x}_{1}(t) \\
\dot{x}_{2}(t) \\
\dot{x}_{3}(t)
\end{array}\right)=\left(\begin{array}{c}
x_{2}(t) \\
-\left(\frac{\mu_{0} N^{2} A}{4 m}\right)\left[\frac{x_{3}(t)}{x_{1}(t)}\right]^{2} \\
-\frac{2 R}{\mu_{0} N^{2} A} x_{1}(t) x_{3}(t)+\frac{x_{2}(t) x_{3}(t)}{x_{1}(t)}
\end{array}\right)+\left(\begin{array}{c}
0 \\
0 \\
\frac{2}{\mu_{0} N^{2} A} x_{1}(t)
\end{array}\right) u(t)
$$

Output equation is $y=x_{1}(t)$, or $z(t)$ position for air-gap control or in the output form.

Linearization of nonlinear electromagnetic suspension EMS system is defined with the suitable stability of an equilibrium point. From Fig. (1), the vertical dynamic of a single degree of freedom of an electromagnetic suspension EMS system is obtained by linearization method. The linearization of force attraction from (15) or (16) around a nominal equilibrium point $\left(i_{0}, z_{0}\right)$ has become by (18) and (19) to [4].

$$
\begin{aligned}
& \mathrm{m} \Delta \ddot{z}(\mathrm{t})=-\frac{\mu_{0} N^{2} A}{4}\left[\frac{i_{0}+\Delta i(t)}{z_{0}+z(t)}\right]^{2}+f_{d}+m g \\
& \mathrm{~m} \Delta \ddot{z}(\mathrm{t}) \cong-\frac{\mu_{0} N^{2} A i_{0}}{2 z_{0}^{2}} \Delta i(t)+-\frac{\mu_{0} N^{2} A i_{0}^{3}}{2 z_{0}^{3}} \Delta z(t)+f_{d}
\end{aligned}
$$

And 


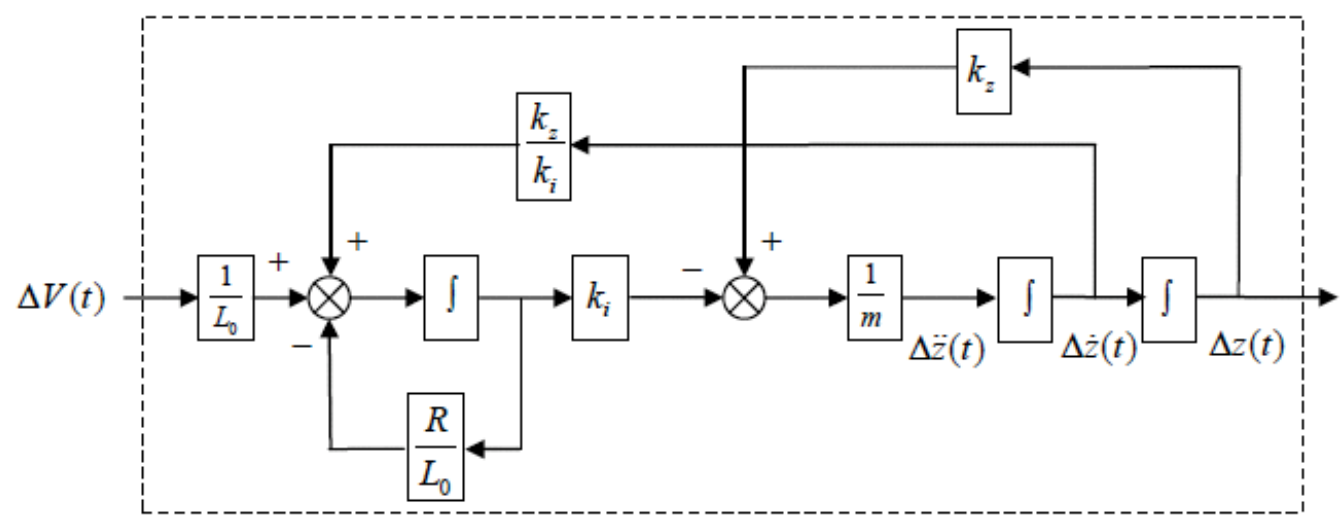

Fig. (2). Linearization model of nonlinear EMS system [2].

$v_{0}+\Delta v(\mathrm{t})=\mathrm{R}\left[i_{0}+\Delta i(t)\right]+\frac{\mu_{0} N^{2} A}{2} \frac{d}{d t}\left[\frac{i_{0}+\Delta i(t)}{z_{0}+z(t)}\right]$

$v_{0}+\Delta v(\mathrm{t})=\mathrm{R} i_{0}+\mathrm{R} \Delta i(t)+\frac{\mu_{0} N^{2} A}{2 z_{0}} \Delta \dot{i}(t)-\frac{\mu_{0} N^{2} A}{2 z_{0}^{2}} \Delta \dot{z}(t)$

The linearization of nonlinear electromagnetic suspension EMS system around the equilibrium point $\left(i_{0}, z_{0}\right)$ with small perturbation is specified for linear electromagnetic suspension EMS model. The linear model is described by open loop of plant model as illustrated in Fig. (2).

$\dot{x}(t)=\left(\begin{array}{ccc}0 & 1 & 0 \\ -\left(\frac{\mu_{0} N^{2} A}{2 m}\right) \frac{i_{0}^{2}}{z_{0}^{3}} & 0 & -\left(\frac{\mu_{0} N^{2} A}{2 m}\right) \frac{i_{0}}{z_{0}^{2}} \\ \left(-\frac{2 R}{\mu_{0} N^{2} A}-\frac{\dot{z}_{0}}{z_{0}^{2}}\right) i_{0} & \frac{i_{0}}{z_{0}} & \left(-\frac{2 R}{\mu_{0} N^{2} A}+\frac{\dot{z}_{0}}{z_{0}}\right.\end{array}\right)\left\{\begin{array}{l}x_{1}(t) \\ x_{2}(t) \\ x_{3}(t)\end{array}\right\}+\left(\begin{array}{c}0 \\ 0 \\ \frac{2}{\mu_{0} N^{2} A} z_{0}\end{array}\right) u(t)$

Where $u(t)=\Delta v(t)$ is defined as input. $\left(i_{0}, z_{0}\right)$ is the prescribed nominal operating point.

\section{OPTIMAL CONTROL}

Performance specification requires to designing EMS system in a large air-gap position control of a non-linear EMS system.

\subsection{Linear Quadratic Regulator (LQR) Design}

Such a given linearization of dynamic model of EMS plant is represented by state-space form in equation (21).

$\dot{x}(t)=A x(t)+B u(t)$

$y(t)=C x(t)$

The state $\mathrm{x}(\mathrm{t})$ is available by measurements. Constructing a controller is to minimize the cost function expressed in equation (22).

$J(u)=\int_{0}^{+\infty}\left(x^{T}(t) Q x(t)+u^{T}(t) R u(t)\right) d t$

Where $Q$ and $R$ are positive definite weighting matrices, for a non zero initial state $x(0)$ at steady state in perturbation of control system, first term in equation (22) subjects to the cost function that is assigned to the "energy" intransient response. And similarly the second term in equation (22) subjects to the cost function that is assigned to the"control energy" used by the controller. Then by choosing weight of matrices $Q$ and $R$, determination of control effort and transient response can be favorable.

The solution to LQR problem is considered the Linear Quadratic Regulation to minimize the cost function. The solution is the linear state feedback control law in equation (23).

$u(t)=K x(t)$

The required state feedback control gain matrix $K$ can be obtained by solving an Algebraic Reccati Equation in equation (24).

$A^{T} P+P A-P B R^{-1} B^{T} P+Q=0$

If $x \in R^{n}$ then this is an $n \times n$ matrix equation and $P$ is the unknown $n \times n$ matrix. If this Riccati equation has a positive definite symmetric solution $P$, then LQR problem has a solution. The required gain matrix is given by equation (25).

$K=-R^{-1} B^{T} P$

The optimal value of the cost function is obtained by equation (26).

$J=x^{T}(0) \operatorname{Px}(0)$

The optimal value of cost function depends on the initial condition $x(0)$.

Stability can be proven by considering the Lyapunov function. From closed loop system state eqaution can be provided by equation (27).

$\dot{x}=\left(A-B R^{-1} B^{T} P\right) x$

The Lyapunove function is expressed by equation (28).

$V(x)=x^{T} P x$

Where $P$ is defined by the Riccati equation by equation (23). Using Riccati equation, the Lyapunov derivative is given by equation (29). 


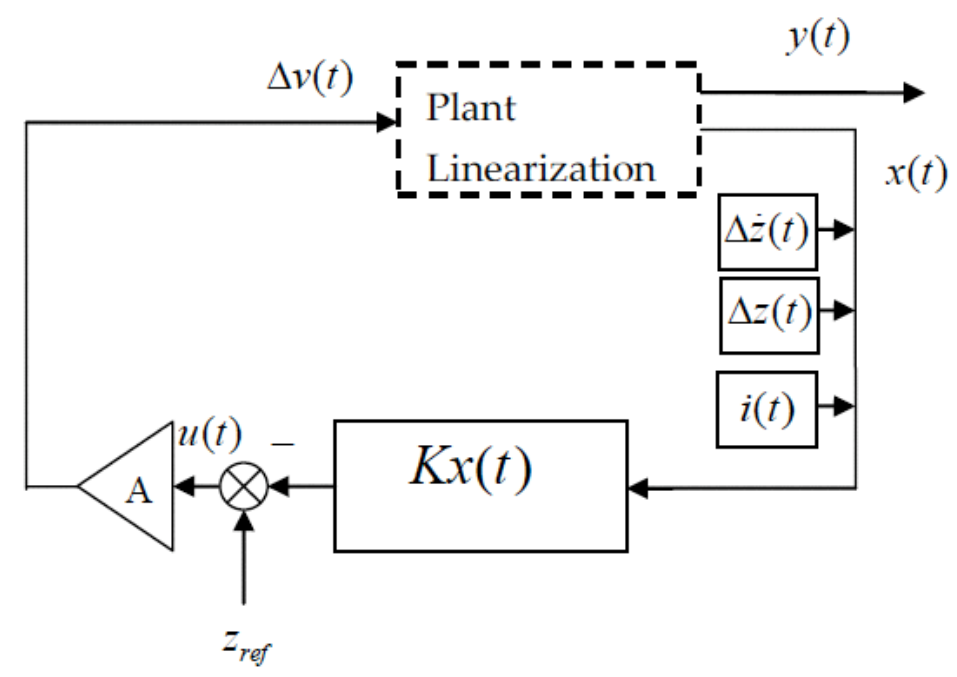

Fig. (3). Overall control structure of nonlinear electromagnetic suspension EMS system.

$\dot{V}(x)=-x^{T}\left\{P B R^{-1} B^{T} P\right\} x-x^{T} Q x$

$\dot{V}(x)<0$

Since the Lyapunov derivative is negative, it concludes that closed loop control system is stable.

The control signal of linear state feedback of $u(t)$ is given in (30).

$u(t)=-\left[k_{p}\left(x_{1}(t)-z_{r e f}\right)+k_{v}\left(x_{2}(t)\right)+k_{a}\left(x_{3}(t)\right)\right]$

Control input $u(t)$ is linearly proportional to air gap or clearance of fixed ferromagnetic track and moving electromagnet coil for stabilize with the prescribed nominal equilibrium air gap at desired operating point $\left(i_{0}, z_{0}\right)$ in design stage. Where $k_{p}$ suppressed the steady-state error, $k_{v}$ controls suspension damping and finally $k_{a}$ taken for overall stability margin, limitation of linear controllers is purely ability to suppress disturbances in control loop. Loop gain in equation (31) must be followed to the bound signal.

$$
L(s)=-K(s I-A)^{-1} B
$$

With the property that

$$
|1+L(j \omega)| \geq 1
$$

For all $\omega$ signal.

The sensitivity function of optimal control system is followed to.

$$
S(s)=\frac{1}{1+L(s)}
$$

It must be satisfied the bound in equation (34).

$$
|S(j \omega)| \leq 1
$$

For all $\omega$ signal.
Fig. (3) shows overall control system of nonlinear electromagnetic suspension EMS system with linearization approximation. The state feedback signals are measured by accelerometer and linear potentiometer sensors. Whereas the velocity is obtained by integral acceleration signal, the air gap position of moving magnet coil is measured by linear potentiometer the set point $z_{\text {ref }}$ with desired air gap control at nominal operating point.

\section{SIMULATION AND EXPERIMENTAL RESULTS}

\subsection{Simulation Results}

The simulation results of controller design of electromagnetic suspension EMS system are illustrated in Fig. (4). This simulation is to design the air gap positioning control of nonlinear electromagnetic suspension EMS system with single degree of freedom between electromagnetic moving coil and fixed ferromagnetic guided track. The linearization of nonlinear electromagnetic suspension EMS system is obtained for simulation as shown in Fig. (2). In this simulation study, linear quadratic regulator is designed to control the EMS system.

From the final state-space model of linear EMS system in equation (20), state matrix can be derived from the numerical parameters at a nominal operating point $z_{0}=0.009 \mathrm{~m}, i_{0}=0.8 \mathrm{~A}$ with neglecting the EMS velocity state $\dot{z}_{0}$. The parameters of design EMS system are followeing; $N=2600, m=3.7 \mathrm{~kg}, A=0.004 \mathrm{~m}^{2}, R=60 \Omega$. The air permeability is equal to $\mu_{0}=4 \pi \times 10^{-7}$.

$$
\dot{x}(t)=\left(\begin{array}{ccc}
0 & 1 & 0 \\
-4029.62 & 0 & -5037.03 \\
-2825.23 & 88.88 & -3531.54
\end{array}\right)\left\{\begin{array}{l}
x_{1}(t) \\
x_{2}(t) \\
x_{3}(t)
\end{array}\right\}+\left(\begin{array}{c}
0 \\
0 \\
0.529
\end{array}\right) u(t)
$$

Output equation is $y=x_{1}(t)$. Linear state-space model and parameters are calculated according to the developed EMS system model described in equation (35). $Q$ is the 


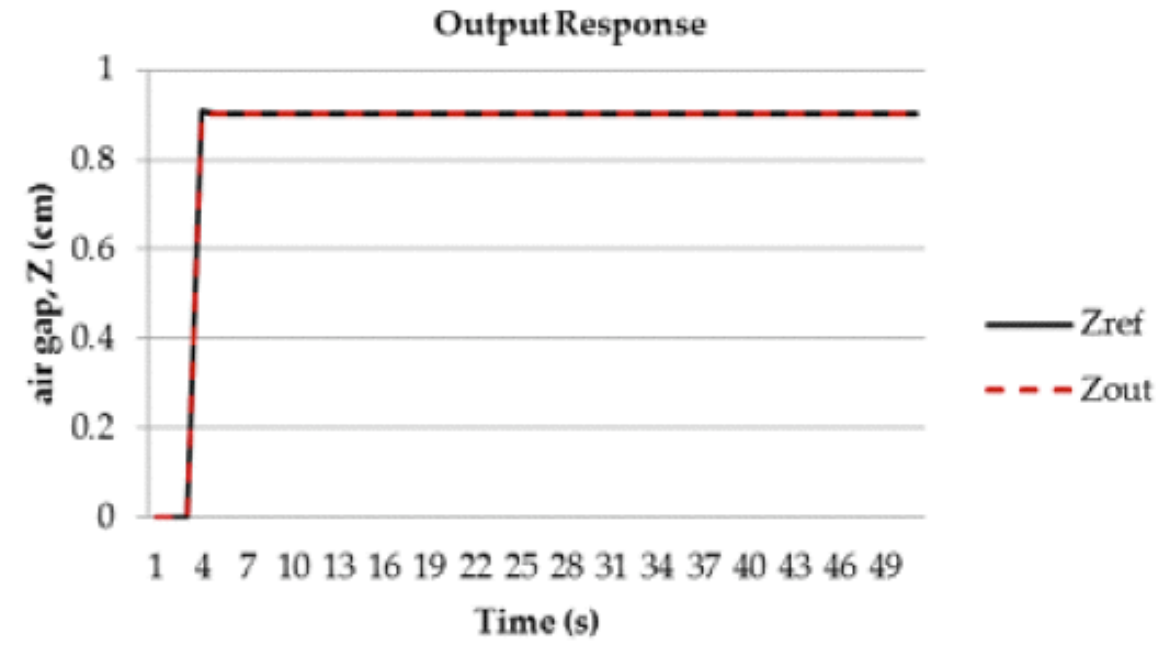

Fig. (4). Set point tracking of air gap control.

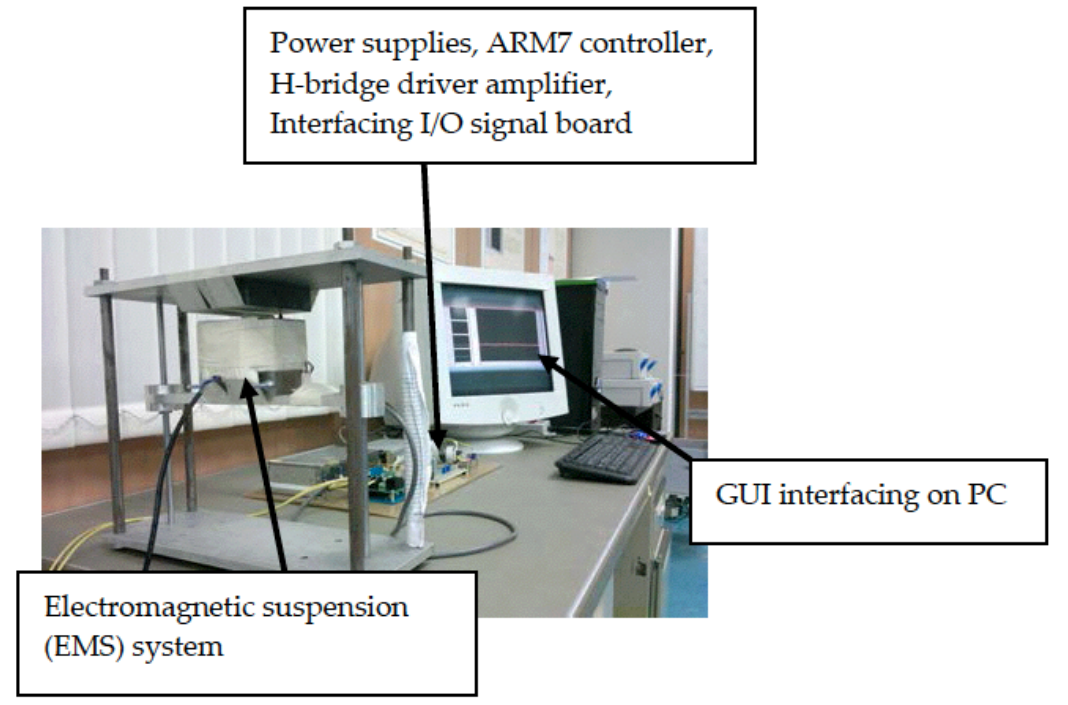

Fig. (5). Experimental set up.

weighting diagonal matrix. $R$ is the weighting matrix, and $P$ matrix from Riccati equation is in equation (36).

$P=\left(\begin{array}{rrr}213.5200 & 1.6795 & -2.3956 \\ 1.6795 & 0.0173 & -0.0245 \\ -2.3956 & -0.0245 & 0.0351\end{array}\right)$

The control gains $K$ matrix which is calculated from equation $(25)$ by $K=\left(\begin{array}{lll}-1.2673 & -0.0130 & 0.0186\end{array}\right)$ in state feedback control. When loop gain is calculated by $L(s)=-0.0076$, thus system is concluded to gaurantee stablility. The output tracking of reference air gap position signal is reached to the reference air gap set point $0.9 \mathrm{~cm}$ as represented in Fig. (8).

\subsection{Experiment Results}

Fig. (5) is illustrates the design of electromagnetic suspension EMS system for testing the proposed control system in laboratory. The material is applied the ferrite permanent magnet type for low reluctance and high permeability for generating the magnetic field. Moving magnetic coil is made by using an iron core with multiple strips compounded in Eshape with dimension of $55 \times 114 \times 76 \mathrm{~mm}$. The wire coil dimension is approximately $90 \times 74 \times 57.7 \mathrm{~mm}$.

In experiment, the control system implementation is represented by block diagram as shown in Fig. (6). The number of turns of moving magnet coil is wound approximately 2,600 turns. Diameter of lead wire is $0.45 \mathrm{~mm}$. Wire lead resistance $(\mathrm{R})$ is about $60 \Omega$. Iron core material uses moving magnet coil. Length of magnet coil is $0.0057 \mathrm{~mm}$. $\mu_{0}$ is equal to $4 \pi \times 10^{-7}$.

Sensors measure the feedback of an air gap position, velocity, and current. Air-gap position is the contact type of resistive potentiometer with range of $0-15 \mathrm{~mm}$. Current signal is applied in the Hall Effect sensor. Range of current is measured between $0-100 \mathrm{~A}$. The velocity signal of moving magnet is derived from the acceleration signal by integral methods. It can provide the range of measurement of acceleration around $\pm 2 g$. All measured sensing signals are fed back to controller. The controller model is selected for 


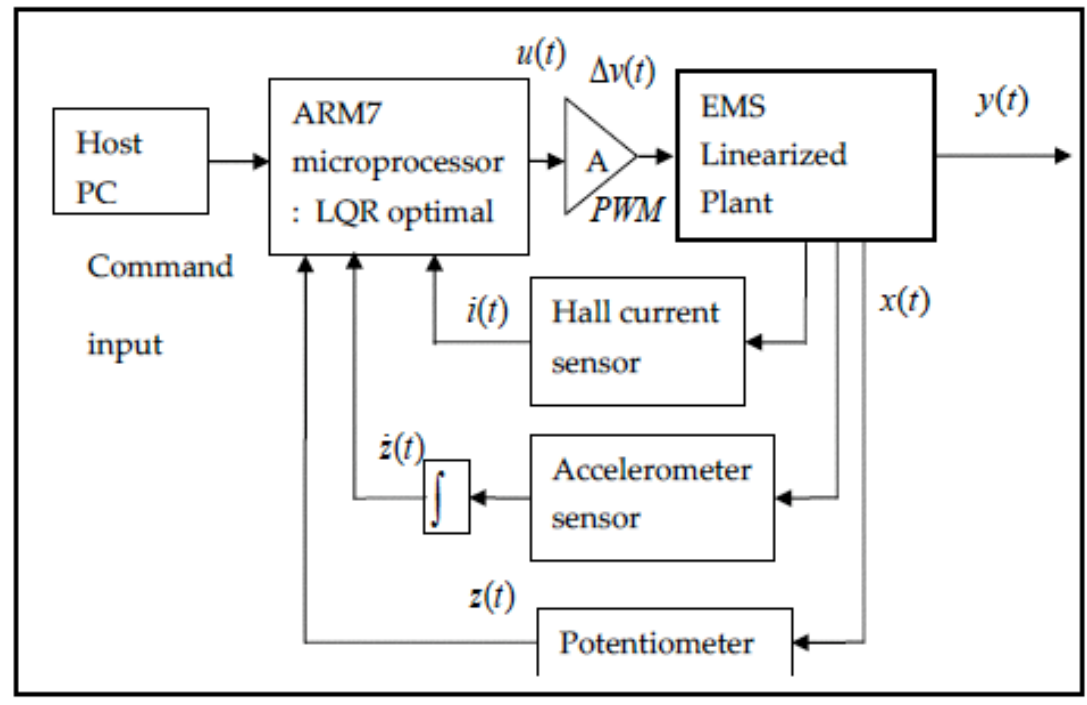

Fig. (6). Control structure in experiment.

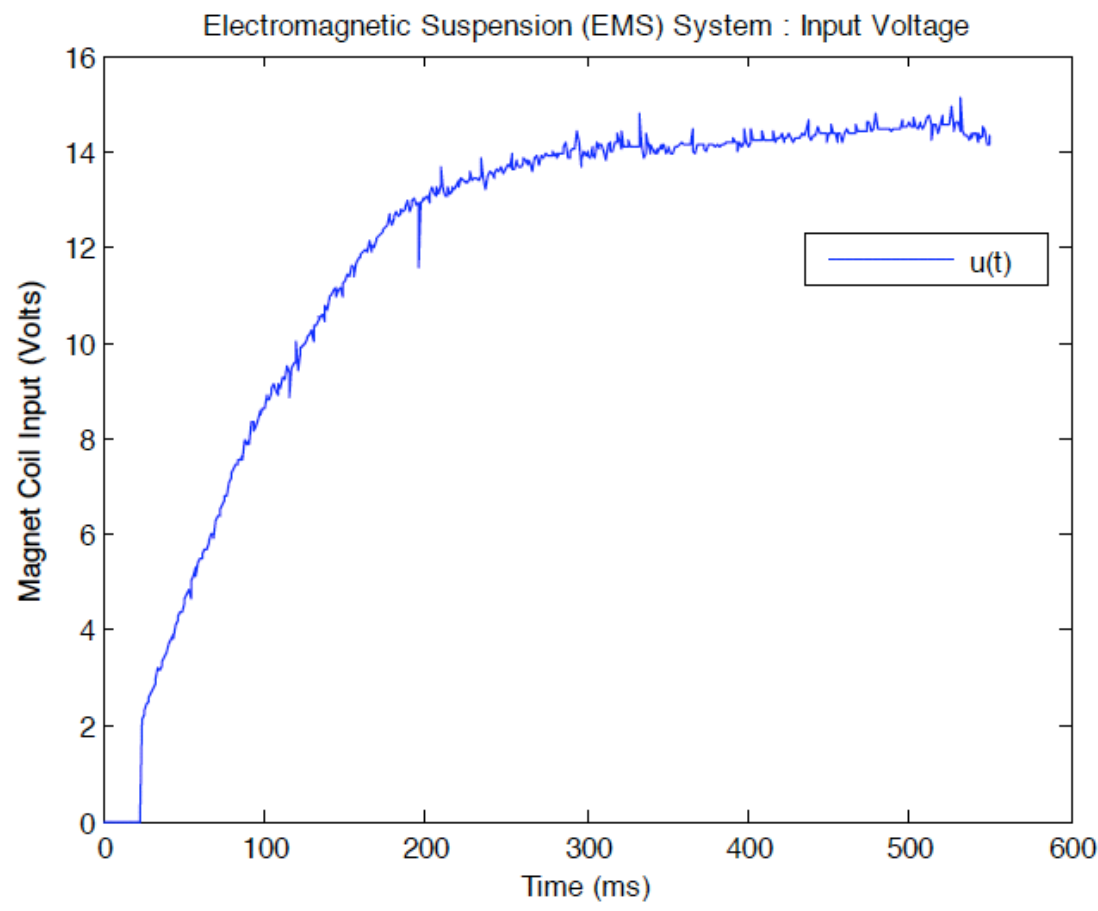

Fig. (7). Relationship of air gap position, set point command, and output voltage.

the LPC2103 ARM7 with 32-bits microcontroller. ADC port is used for interfacing the feedback sensing signals. The control output signal of ARM7 controller is sent in Pulse Width Modulation (PWM) signal in range of $0-3.3 \mathrm{mV}$ to amplifier. H-bridge amplifier type can supply the maximum voltage and current about $24 \mathrm{Vdc}$ and $80 \mathrm{~A}$ respectively. The $\mathrm{H}-$ bridge amplifies the signal in range of $0-24 \mathrm{Vdc}$ to magnet coil. Initial position of moving magnet coil which is measured from fixed ferromagnetic guided track as the starting point of an experiment is placed with distance about 11.7 $\mathrm{mm}$ in vertical direction. Fig. (7) shows the relationship of air gap position, set point command, and output voltage signals. From the result of experiment, the output voltage from controller generates the PWM signal to moving magnet coil by $14 \mathrm{Vdc}$ with equal to the desired air gap position at 9.5 mm measured from fix ferrite permanent magnet guided track. The set point is set from the GUI from personal computer which is $9.5 \mathrm{~mm}$ the output of moving magnet can track the reference of air-gap position as shown the response time in Fig. (8).

In summary the nonlinear electromagnetic suspension EMS system control can be controlled by using linear quadratic regulation with state feedback control method. The air gap position of electromagnetic moving coil and fixed ferromagnetic guided track can be verified by experiment. Air gap position with equilibrium point at $9.5 \mathrm{~mm}$ can be controlled, but the system is very low response due to its nonlinear characteristic of EMS system. 


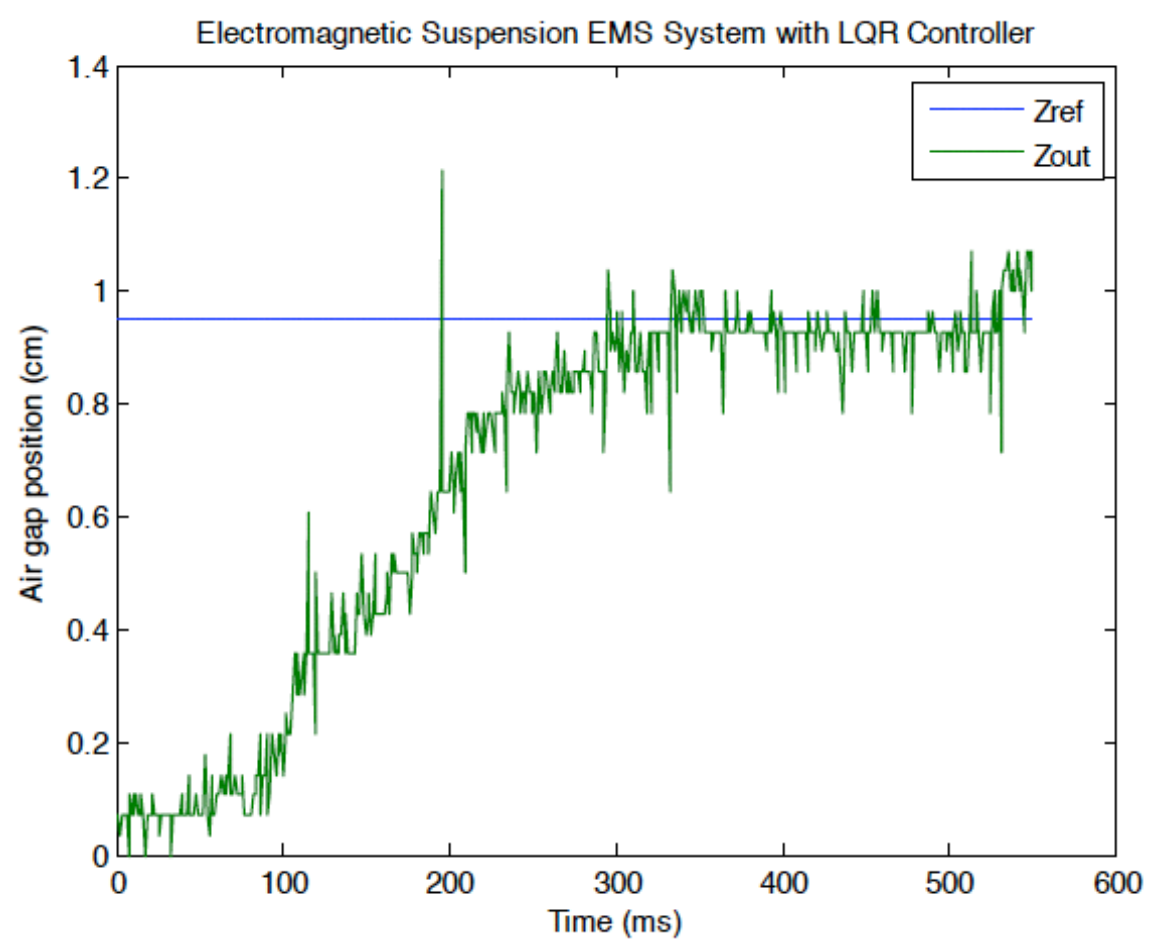

Fig. (8). Response of air gap position control with $9 \mathrm{~mm}$ for experiment.

\section{CONCLUSION}

This research proposed the control design of a nonlinear electromagnetic suspension EMS system. Linear state feedback control by using linear quadratic control of air gap position is designed. In conclusion, the system performance of linear state feedback control using linear quadratic regurator (LQR) control can be applied for controlling the nonlinear electromagnetic suspension EMS system as shown in simulation and experiment. The system stability and adaptation are guaranted by using Lyapunov's function candidate. The proposed control performance is robustly stable.

\section{CONFLICT OF INTEREST}

The author confirms that this article content has no conflicts of interest.

\section{ACKNOWLEDGEMENTS}

The research described in this paper was partially supported by the Ministry of University Affairs (MUA), Thailand.

\section{REFERENCES}

[1] P. K. Sinha and A. N. Pechev, " Nonlinear controller for electromagnetic suspension systems," International Journal of Advanced Robotic Systems, vol. 7, pp. 27-40, 2010.

[2] Z. Zhang, Z. Long, L. She and W. Chang, "linear quadratic state feedback optimal control against actuator failures," in 2007 IEEE International Conference on Mechatronics and Automation, Habin, 2010, pp. 3349-3354.

[3] S. J. Joo and J. H. Seo, " Design and analysis of the nonlinear feedback linearizing control for an electromagnetic suspension system," IEEE Transactions on Control Systems Technology, vol. 5, pp. 2740, 1997.

[4] P. K. Sinha, Electromagnetic Suspension: Dynamics \& Control. London, U.K.: Peter Peregrinus, 1987.

[5] W. G. Hurley, M. Hynes, and W. H. Wölfle, " PWM control of a magnetic suspension system," IEEE Transactions on Education, vol. 47 , pp. 165-173, 2004

[6] C-S. Lin, Y-L. Lay, P-W. Chen,Y-J. Jain, and S-W. Chen, "The laser displacement measurement with feedback control in a magnetic levitation and suspension system," Computer Methods in Appied Mechanic and Engineering, vol. 190, pp. 25-34, 2000.

[7] T. Gluck, W. Kemmetmuller, C. Tump, \& A. Kugi, "A novel robust position estimator for self-sensing magnetic levitation systems based on least squares identification", Control Engineering Practice, vol. 19, pp. 146-157, 2011.

Received: June 04, 2013

(C) Anan Suebsomran; Licensee Bentham Open.

This is an open access article licensed under the terms of the Creative Commons Attribution Non-Commercial License (http://creativecommons.org/licenses/by-nc/3.0/) which permits unrestricted, non-commercial use, distribution and reproduction in any medium, provided the work is properly cited. 